\title{
Measurement of activity in small animals using a microcomputer-controlled system
}

\author{
ROBERT W. SILVERMAN, ANN S. CHANG, and ROGER W. RUSSELL \\ University of California, Los Angeles, California
}

\begin{abstract}
A computer-controlled system that detects spontaneous activity (locomotion and rearing) and diurnal activity rhythms in small animals is described. Automatic recording of subtotals during test periods provides data about habituation (nonassociative learning). The system is relatively inexpensive to construct. Fabricated from highly reliable circuit components, it provides replicable measures that enable comparisons between different experimental treatments to be made using parametric statistics.
\end{abstract}

During recent years, studies of the effects on living organisms of manipulating neurotransmitter or neuroendocrine systems have revealed that it is important to measure individual differences in spontaneous activity. Spontaneous activity is defined as movements in familiar or novel environments in which no special stimulus conditions are introduced, as opposed to activity induced by specific environmental stimulation (Silverman, Chang, \& Russell, 1988). Typical of spontaneous activity in normal animals under many experimental treatments is a decrease in magnitude (habituation) as the duration or frequency of exposure increases; under other treatments, such a change does not occur. Also subject to change as conditions vary is the diurnal (circadian) rhythm that characterizes activity during the normal daily light:dark cycle. The need to differentiate and to measure these behavioral properties has become especially urgent as efforts have continued toward the development of valid animal models of such human states as hyperkinesia, the affective disorders, degenerative dementias (e.g., Alzheimer's disease), and behavioral changes during aging. Reiter and MacPhail (1979) and, more recently, Clarke, Smith, and Justesen (1985) have discussed the advantages and limitations of different techniques for measuring activity. The present paper describes a computer-controlled system that provides information about the processes of habituation and diurnal rhythm.

Figure 1 presents a block diagram of the activity monitor system. The apparatus monitors spontaneous activity for 5 rats simultaneously. Each of five chambers is equipped with solid state infrared emitter and detector arrays that monitor changes of an animal's position by sens-

The apparatus described in this paper was developed with the support of USPHS Grant MH17691 to Donald J. Jenden, whose helpful comments during the design and preparation of this report we wish to acknowledge. We also thank Ruth Booth and Sharlene Lauretz for their assistance in obtaining the data presented, Holly Batal for preparation of the manuscript, and James Randklev for photography. Correspondence may be addressed to the authors at Department of Pharmacology, School of Medicine and the Brain Research Institute, University of California, Los Angeles, CA 90024-1735. ing the breaks in infrared light beams. The circuitry has been constructed for use with an IBM XT personal computer or a Radio Shack TRS-80 Model III microcomputer that collects the data and performs the necessary analysis from all five chambers.

Each chamber (Figure 2) consists of a thin-walled, aluminum cylinder with a plywood top cover (not shown), and is set on a wood frame covered with wire screen. The cylinder is fabricated from a single piece of $16 \mathrm{ga}$ (1.56 mm thick) sheet aluminum, so that the dimensions are $60 \mathrm{~cm}$ in diameter and $30 \mathrm{~cm}$ in height. The circular shape was chosen to eliminate the possibility of the animal's finding a "safe corner" that would reduce spontaneous locomotion. Two arrays of infrared light-emitting diodes (IRLEDs) and photodarlington transistor detectors are attached to the walls of the chambers (Figure 3A). A rectangular array of $3 \times 3$ is situated $4 \mathrm{~cm}$ above the floor of the chamber to register horizontal movements (Figure 3B). A second array of $5 \times 5$ is located $10 \mathrm{~cm}$ above the floor to monitor vertical movements (Figure 3C). The increased density of the second array is required to pick up movements of the subject's head or nose, which presents a smaller surface to occlude an infrared beam than does the animal's body to the lower, $3 \times 3$ array. The cylindrical chamber rests on a $66-\mathrm{cm}$ square wood frame covered with a wire screen (4 mesh per linear inch) under which are wood shavings. Each IRLED and phototransistor is mounted in a black acrylic plastic block at the end of a hole (approximately $20 \mathrm{~mm}$ deep) that functions as a collimating tube (Figure 3D). This greatly decreases both the dispersion of the emitter beam and the angular response of the detector. Both devices, as supplied, are equipped with lens windows to focus the beam. Further reduction in dispersion and detection angles is produced by the mounting technique, which permits the relatively close spacing of beams in the apparatus. The large distance between detector and emitter requires the use of a high-intensity source (TEMT8850, Three-Five Systems, Phoenix, AZ) that produces a minimum infrared light output of $50 \mathrm{~mW} / \mathrm{ster}$ adian. Similarly, a sensitive detector that has a ratio of 


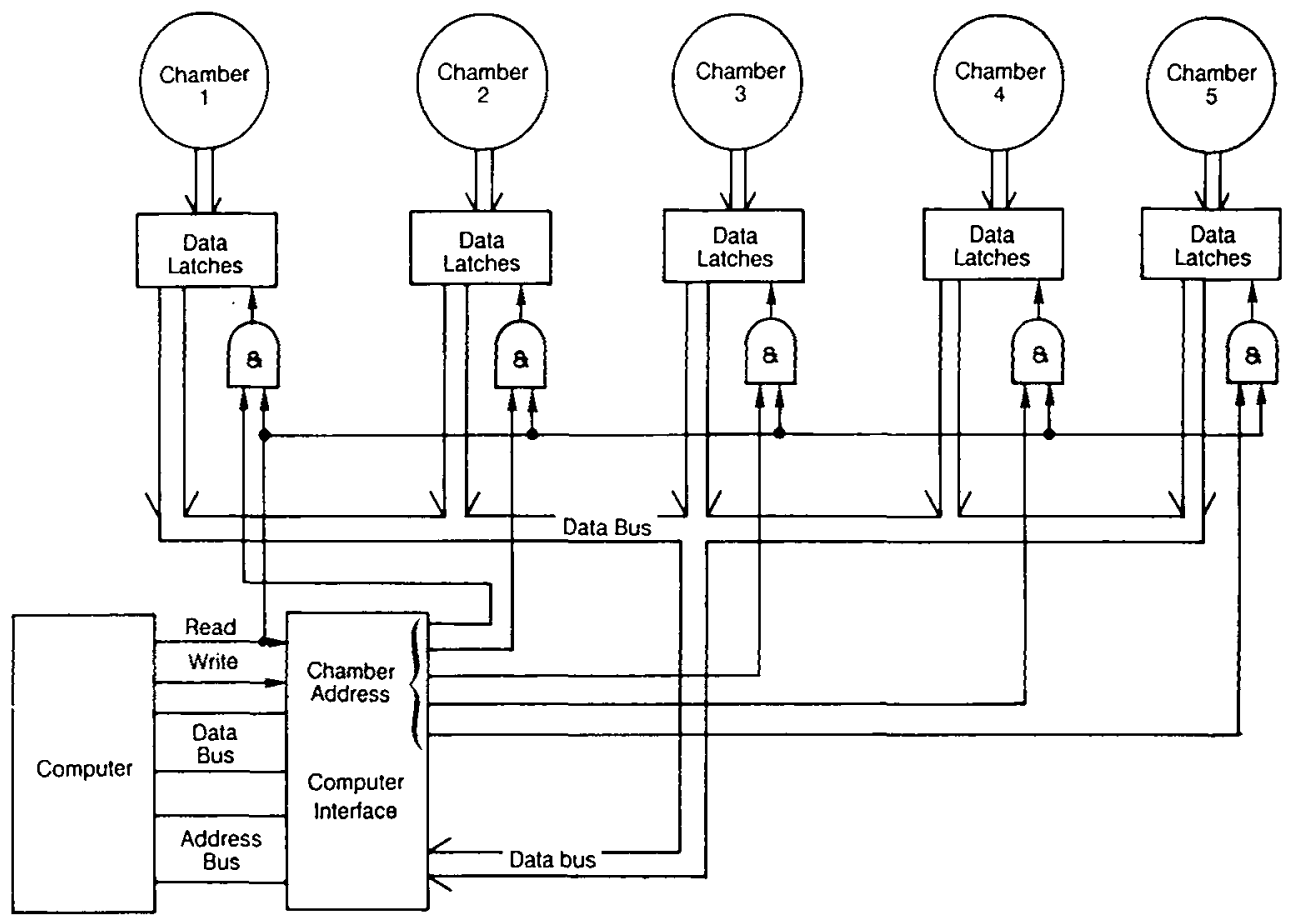

Figure 1. Block diagram of the system for measuring spontaneous activity.

light to dark current of $>10^{4}$ (CLR 2180, Clairex Electronics, Mt. Vernon, NY) is employed. The chambers are located in a dark room and are under dim red light throughout all trials. Control of the auditory environment is achieved by the use of a white-noise source.

Each chamber is equipped with a separate power supply for the IRLEDs and contains circuitry to generate logic-level signals $(>3.5 \mathrm{~V}$ with no beam interruption and $<0.4 \mathrm{~V}$ when a beam is broken) (Figure 4). The IRLEDs are operated at a continuous current of $100 \mathrm{~mA}$

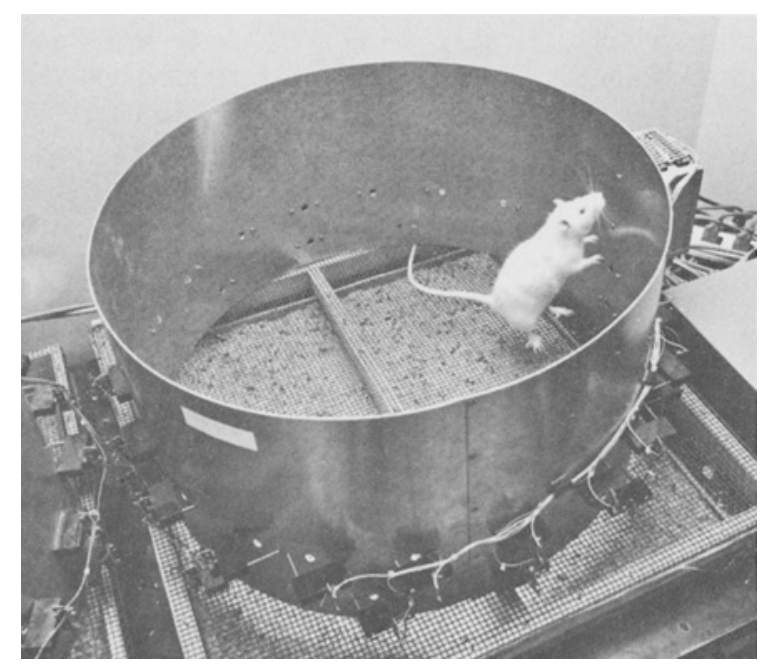

Figure 2. Photograph of the activity test environment, showing one of five chambers. each. Each of the six phototransistors for sensing horizontal movements provide a separate output signal that is conditioned by one hex, Schmitt trigger inverter (74LS14) and fed to six single-bit data latches located in the interface box. By reading the data from the six data latches, both total horizontal movements and horizontal position can be monitored. The data stored in the six latches are summed by the computer program to provide the measure of total horizontal movement. After passing through a Schmitt trigger inverter, the signals from each of the 10 phototransistors monitoring vertical activity are OR'ed together, and drive a single data latch in the interface to record rearing.

The interface box contains the necessary data, address, and control line buffers to allow connection directly to the input-output $(\mathrm{I} / \mathrm{O})$ lines of the microcomputer and the five sets of seven data latches. An $8255 \mathrm{I} / \mathrm{O}$ controller integrated circuit controls the interface electronics. By sending a chamber select address to the interface box and then reading the contents of the seven latches, the data from a chamber can be obtained. Approximately $10 \mu \mathrm{sec}$ after the read operation, the latches are automatically reset. The interface box circuitry also generates a reset pulse upon power turn-on to initialize all of the data latches. An 8255-based I/O board for the IBM microcomputer used to control and read out the latches may be purchased from numerous manufacturers (e.g., Industrial Computer Source, San Diego, CA, Model PC-DIO24), or the investigator may construct the circuitry (see Sargent \& Shoemaker, 1984). 
Before an experimental trial, the operation of each chamber is checked by sequentially blocking each infrared beam with an opaque object to see that the computer registers the break.

Two programs, written in the $\mathrm{C}$ computer language, allow an IBM personal computer to control and collect data from the activity monitor (similar programs written in a combination of BASIC and Assembler are available for the TRS-80 Model III microcomputer). Program AM measures spontaneous activity by summing inputs from the horizontal data latches and the vertical (rearing) data latch over equal length intervals. Program AM24 records total activity over a 24-h period, during which data are initially summed over short intervals to allow observation of possible habituation and then, for the remainder of the experiment, measurements are made over much longer intervals. Except for the formatting of the final output, both programs are very similar and share the same data collection routine. In both programs, a trial is divided into a user-specified number of time intervals, each of a user-specified duration. Two important variables are $V$, a two-dimensional array that holds the number of verti-

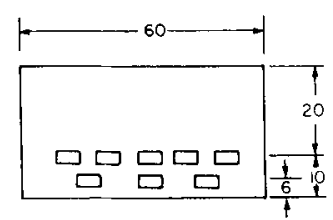

$A$

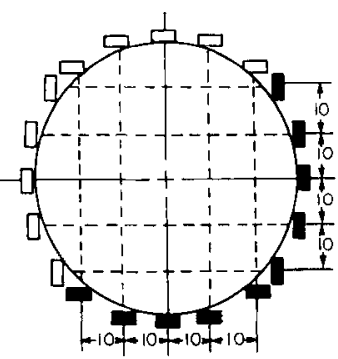

C

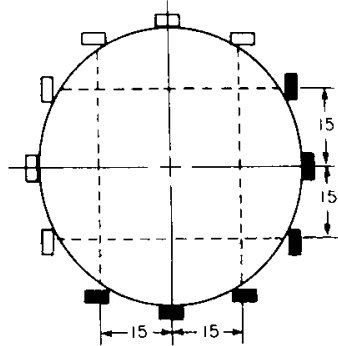

B

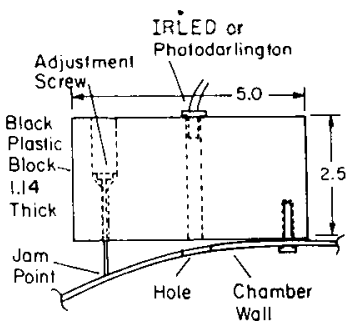

D
Figure 3. Diagram of the activity test environments (chambers), with locations of the infrared light-emitting diodes (open rectangles) and the photodarlington transistor detectors (solid rectangles). A is a side view of the chamber. $B$ is a top view showing the horizontal motion array and $C$ shows the "rearing" array. $D$ is a diagram of the black plastic mounting block used for all of the infrared lightemitting diodes and photodarlington sensors. All dimensions are in centimeters.

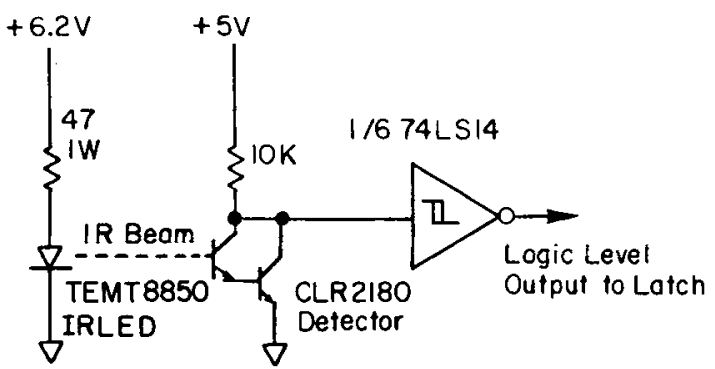

Figure 4. Circuit diagram of one of 16 identical infrared lightemitting diode and phototransistor pairs.

cal movement sensor triggers for each interval for each chamber, and $\mathrm{H}$, a two-dimensional array that holds the horizontal movement sums for each interval for each chamber.

After printing a title on the screen, the AM program asks the user for the number of chambers, the number of intervals, and interval length. The program waits until the user types a code indicating that the equipment and animals are ready, then prints headings for the video display into a window at the top of the display so that they cannot be overwritten by subsequent output, and initializes the $8255 \mathrm{I} / \mathrm{O}$ controller. The data collection routine then is called.

The data collection routine collects, stores, and displays the data from the activity monitor interface. To start, it zeros the $\mathrm{V}$ and $\mathrm{H}$ arrays, resets the beam sensor data latches by reading the input word for each of the five chambers, and then synchronizes the routine to the seconds counter of the computer system clock. Next, the routine enters its main loop. It first checks if the user pressed the Q key (Quit) to stop the data collection before the end of the trial. If so, the number of intervals completed and program control are returned to the calling routine. The seconds counter then is checked. If the end of an interval has been reached, the number of vertical and horizontal movements is saved into the appropriate arrays, and a new line is set up on the video display for the results of the next interval. If the trial is done (all the intervals completed), the number of intervals completed and program control are returned to the calling routine. Otherwise, the movement sensor data latch outputs for each of the chambers are read in turn, which subsequently resets the data latches. The appropriate sums for this interval are updated in the data arrays ( $\mathrm{V}$ and/or $\mathrm{H}$ ) and on the video display. This completes one pass through the main loop, and the next can be started. On an IBM PC, this routine can read each chamber 16.3 times per second, even when all latch bits are set (this condition requires maximum processing).

After the data collection is complete, the AM program prints the number of intervals completed and then asks the user how many subtotals to print. This allows the user to collapse the number of data points printed. For example, if the trial consisted of 302 -sec intervals, the user 
ACTIVITY MONITOR 07/10/87 04:09:04

AMINODRUN7

SUMMING INTERVAL LENGTH $=120$ SECONDS NUMBER OF SUMMING INTERVALS PER SURTOTAL 1

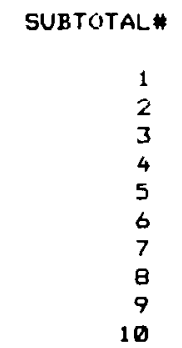

GRAND TOTAL

$\begin{array}{cr}\text { BOX } & 1 \\ V & H \\ 32 & 125 \\ 16 & 51 \\ 44 & 87 \\ 23 & 103 \\ 21 & 44 \\ 27 & 61 \\ 30 & 28 \\ 17 & 56 \\ 16 & 25 \\ 15 & 26\end{array}$

$241 \quad 606$

$\begin{array}{cr}\text { BOX } & 2 \\ V & H \\ 40 & 112 \\ 44 & 146 \\ 33 & 110 \\ 27 & 130 \\ 30 & 84 \\ 36 & 95 \\ 28 & 52 \\ 19 & 79 \\ 30 & 71 \\ 39 & 78\end{array}$

$326 \quad 957$

$\begin{array}{rr}\text { ROX } & 3 \\ V & H \\ 25 & 55 \\ 105 & 194 \\ 46 & 89 \\ 61 & 127 \\ 37 & 67 \\ 44 & 74 \\ 29 & 51 \\ 20 & 38 \\ 25 & 77 \\ 23 & 37\end{array}$

$415 \quad 809$

\begin{tabular}{cccc} 
BOX & \multicolumn{2}{c}{ BOX } & \multicolumn{2}{c}{$\mathrm{V}$} & $H$ \\
$V$ & $H$ & 25 & 113 \\
21 & 119 & 25 & 88 \\
25 & 120 & 23 & 92 \\
28 & 117 & 29 & 92 \\
15 & 72 & 27 & 95 \\
14 & 77 & 14 & 42 \\
15 & 76 & 29 & 78 \\
22 & 80 & 15 & 64 \\
7 & 44 & 9 & 34 \\
19 & 45 & 22 & 45 \\
18 & 49 & 11 & 39
\end{tabular}

$184 \quad 799 \quad 204 \quad 690$

Figure 5. Reproduction of the activity monitor output for a control animal.

could choose to print all the data ( 30 subtotals) or to print the data summed over 6-sec intervals (10 subtotals). A title and heading are printed, and then the vertical and horizontal movement counts for each chamber are printed for each subtotal interval (Figure 5). The user can print the same data many times with different subtotals. Once the results have been printed to the user's satisfaction, a new trial can be started. The data, thus recorded, provide the two major measures in which we are interested: the sums of data during the total trial is taken as the measure of spontaneous activity in each of the two planes, and subtotals over the trial give us a basis for studying habituation, as shown in Figure 6, for rearing behavior.

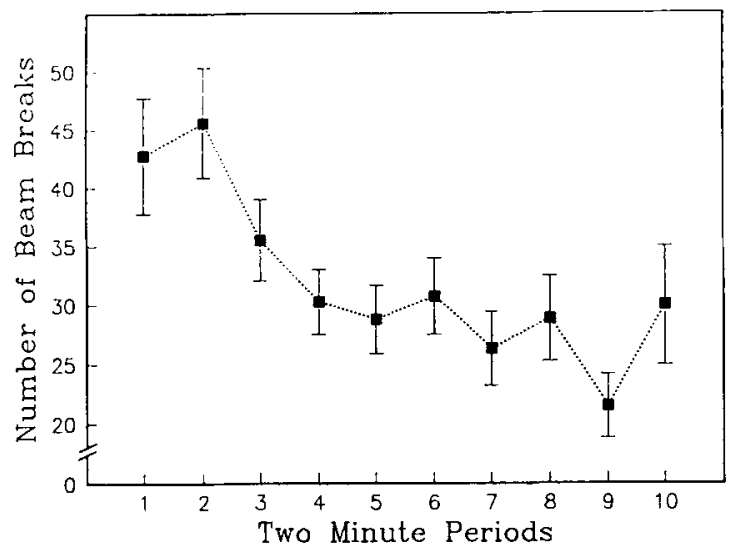

Figure 6. Plot of means of vertical activity (rearing) during a 20min test session. Habituation is exhibited after the initial response to the novel situation.
The AM24 program uses the same variables and has the same general structure as the AM program; however, it repeats the data-collection part of the AM program, using a different interval length and number of intervals each time. Currently, the first pass consists of 152 -min intervals and the second pass of 4730 -min intervals. When the data collection routine is called with the appropriate elements of the $\mathrm{H}$ and $\mathrm{V}$ arrays, the data for the second pass are stored in the arrays $\mathrm{H}$ and $\mathrm{V}$ after the data for the first pass. At the end of the experiment, the activity levels for the first half hour are printed on one page, with the results for the rest of the day on the next page.

Availability. A copy of the computer program is available for the IBM or Radio Shack microcomputers by sending a formatted diskette to the authors.

\section{REFERENCES}

Clarke, R. L., Smith, R. F., \& Justesen, D. R. (1985). An infrared device for detecting locomotor activity. Behavior Research Methods, Instruments, \& Computers, 17, 519-525.

Reiter, L. W., \& MacPhall, R. C. (1979). Motor activity: A survey of methods with potential use in toxicity testing. Neurobehavioral Toxicology, 1(Suppl. 1), 53-66.

SARGent, M., III, Shoemaker, R. L. (1984). The IBM Personal Computer from the inside out. Reading, MA: Addison-Wesley.

Silverman, R. W., Chang, A. S., \& Russell, R. W. (1988). A microcomputer-controlled system for measuring reactivity in small animals. Behavior Research Methods, Instruments, \& Computers, 20, 495-498.

(Manuscript received February 4, 1988; revision accepted for publication June 8, 1988.) 